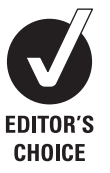
College of Medicine, Chungbuk National University, Cheongju, Korea

${ }^{2}$ Department of Pediatrics, Samsung Medical Center, Sungkyunkwan University School of Medicine, Seoul, Korea

${ }^{3}$ Department of Pediatrics Chung-Ang University Yongsan Hospital, Yongsan, Seoul, Korea

\section{Correspondence to}

Yon Ho Choe, Department of Pediatrics, Samsung Medical Center, Sungkyunkwan University School of Medicine, 50 Irwon-dong, Gangnam-gu, Seoul 135-710, South Korea: i101016@skku.edu

Accepted 1 August 2010 Published Online First 23 September 2010

\title{
The effects of three alternative treatment strategies after 8 weeks of proton pump inhibitor therapy for GERD in children
}

\author{
Ji Hyuk Lee, ${ }^{1}$ Mi Jin Kim, ${ }^{2}$ Jong Seung Lee, ${ }^{3}$ Yon Ho Choe ${ }^{2}$
}

\begin{abstract}
Objectives The purpose of this 24-week treatment study was to evaluate the effects of three treatment strategies after 8 weeks of lansoprazole therapy for gastroesophageal reflux disease in children.
\end{abstract}

Design Open-labelled, uncontrolled, prospective study. Setting Samsung Medical Center, Seoul, Korea. Methods 37 erosive reflux disease (ERD) and 20 non-erosive reflux disease (NERD) patients were divided into three groups by symptom assessment at 8 weeks: (1) observation without treatment in the 'symptoms-resolved' group, (2) 'on-demand' treatment for an additional 16 weeks in the 'symptomsattenuated' group and (3) continuous treatment in the 'symptoms-persistent' group.

Results For ERD, six (100\%) out of six patients in the 'symptoms-resolved' group remained improved at weeks 16 and 24 . Sixteen (72.7\%) out of 22 patients in the 'symptoms-attenuated' group had improvement of symptoms at 16 weeks, and 18 (81.8\%) patients at 24 weeks. Six $(66.7 \%)$ out of nine patients in the 'symptoms-persistent' group remained improved at weeks 16 and 24. For NERD, seven (100\%) out of seven patients in the 'symptoms-resolved' group remained improved at weeks 16 and 24 . Eight (80.0\%) out of 10 patients in the 'symptoms-attenuated' group remained improved at week 16 , and 10 (100.0\%) patients at week 24 . None out of three patients in the 'symptoms-persistent' group remained improved at weeks 16 and 24.

Conclusions The selection of each alternative for long-term management according to the results of the assessment of symptoms at week 8 was useful and well tolerated. 'On-demand' therapy was equally effective. The 16-week therapy had the same efficacy as the 24-week therapy with regard to long-term lansoprazole treatment.

\section{INTRODUCTION}

Gastroesophageal reflux disease (GERD) is a condition that develops when reflux of the stomach contents causes irritating symptoms and/or complications. Erosive reflux disease (ERD) is defined by the presence of troublesome symptoms caused by the reflux of gastric contents and the presence of mucosal breaks during endoscopy. Non-erosive reflux disease (NERD) is defined by the presence of irritating reflux-associated symptoms and the absence of mucosal breaks during endoscopy. ${ }^{1-5}$ The efficacy of proton pump inhibitor (PPI) treatment in adult patients with GERD has been confirmed by

\section{What is already known on this topic}

Although there are a limited number of paediatric studies on the treatment of gastroesophageal reflux disease (GERD) with proton pump inhibitor therapy, the effect is reported to be similar to that in adults. However, data and consensus on long-term PPI treatment in children with GERD are lacking.

\section{What this study adds}

The selection of each alternative for long-term management according to the results of the assessment of symptoms at week 8 was useful and well tolerated in children. 'On-demand' therapy was equally effective. The 16-week therapy had the same efficacy as the 24-week therapy with regard to long-term lansoprazole treatment.

many studies. ${ }^{6-10}$ Although there are a limited number of paediatric studies on the treatment of GERD with PPI therapy, the effect is reported to be similar to that in adults. ${ }^{11-14}$

For maintenance treatment with PPI, there are different long-term management strategies that have been reported in the literature; These include various 'step-down' approaches such as changing to a cheaper histamine2-receptor antagonist, PPI therapy taken on alternate days or 'on-demand' and a reduced dose of PPI. ${ }^{15}$ However, data and consensus on long-term PPI treatment in children with GERD are lacking.

Therefore, a prospective study using treatment strategies according to the presence or absence of symptoms after 8 weeks of PPI therapy was conducted. The strategies included observation without treatment in a group with complete resolution of symptoms, 'on-demand' treatment for an additional 16 weeks in a group with partial improvement and continuous treatment without dose reduction for 16 weeks in a group with no improvement. The purpose of this 24 -week treatment study was to evaluate the effects of the three alternative treatment strategies after 8 weeks of PPI therapy for GERD in children. 


\section{MATERIALS AND METHODS Patients}

From April 2008 to September 2009, patients were recruited from children aged 4-18 years with gastroesophageal reflux symptoms, such as heartburn, vomiting, regurgitation, sour taste, chronic cough or epigastric pain or epigastric discomfort, who visited the outpatient clinic of the Department of Pediatrics, Samsung Medical Center. Patients with ERD $(\mathrm{N}=43)$ or NERD $(\mathrm{N}=34)$ diagnosed by endoscopy were enrolled. The criteria for exclusion were gastric or duodenal ulcers, food allergy, previous use of PPI, oesophageal stenosis, acute upper gastrointestinal bleeding, physicochemical oesophageal injury, previous oesophageal and/or gastric surgery, oesophageal varix, eosinophilic esophagitis and Helicobacter pylori infection. All patients took no medicine for at least 2 weeks before the study was started. Approval for the study was obtained from the Institutional Review Board at Samsung Medical Center. All participants and/or their guardians provided written informed consent.

\section{Methods}

Treatment strategy

This study was an open-labelled, uncontrolled, prospective, single centre study. The subjects were divided into two groups: ERD and NERD. The patients were treated with lansoprazole at a dose of $15 \mathrm{mg}$ (body weight below $30 \mathrm{~kg}$ ) or $30 \mathrm{mg}$ (body weight over $30 \mathrm{~kg}$ ) once daily. After 8 weeks of treatment, the symptoms were evaluated. Those patients that refused to take the medicine for more than 2 weeks because of improved symptoms were categorised as the 'no more treatment' group. After 8 weeks of treatment, the patients were divided into three subgroups: 'symptoms-resolved', 'symptoms-attenuated' and 'symptoms-persistent' according to their response to the treatment. The treatment was discontinued in the 'symptoms-resolved' group. The patients in the 'symptoms-attenuated' group were treated with 'on-demand' therapy. The patients that showed persistent symptoms were maintained with PPI treatment without dose reduction for an additional 16 weeks. At 16 and 24 weeks, changes in symptoms were reassessed. Simultaneously, a telephone administered questionnaire, for the evaluation of the symptoms, was performed in patients that were lost to follow-up after 2 weeks of treatment.

\section{Follow-up}

Follow-up evaluation was carried out at week 8, 16 and 24. Adverse events associated with the medication, patient compliance and symptoms were evaluated at every visit. At the onset of treatment, patient information and reflux-associated symptoms were recorded at the study interview by the investigator. The patients or parents were educated on how to record the symptoms in their daily diary, which included the characteristics and frequency of pain, severity of symptoms, unspecified symptoms, medication schedule and adverse effects of the medication.

Symptom severity was graded as none $=0$ (asymptomatic), mild=1 (bothered a little or symptoms present part of day or night but causing little or no discomfort and not interfering with sleep), moderate $=2$ (bothered a lot or symptoms present most of the day or night or interfering with daily routine or sleep) or severe $=3$ (bothered intensely or constant symptoms or marked interference with daily routines or sleep). ${ }^{11}$
Symptom assessment

Using the information from the daily diary and interview, the following groups were defined: 'symptoms-resolved' with symptom score improvement over $50 \%$, 'symptomsattenuated' with symptoms improved less than $50 \%$ and 'symptoms-persistent' where the symptoms still persisted. The patients with 'symptoms improved' included those with 'symptoms-resolved' and 'symptoms-attenuated'.

\section{Endoscopy}

Endoscopies were performed in all enrolled patients at week 0 and repeated in the patients with GERD who provided consent at 8 weeks. The endoscopic findings were assessed using the Los Angeles classification (table 1). ${ }^{16}$ During the endoscopy, four biopsies were taken from the distal esophagus (two pieces in each 2 and $5 \mathrm{~cm}$ above the $z$-line) to rule out any disease included in the criteria for study exclusion. Simultaneously, two biopsies were taken from the stomach to rule out $H$. pylori infection.

\section{Statistical analysis}

For the categorical variables, the $\chi^{2}$ test was used as appropriate. For the continuous variables, the Kruskal-Wallis H test was used to compare the three or four groups of cases. $p$ Values $<0.05$ were considered to indicate statistical significance. Statistical tests were performed with SPSS for Windows software V.14.0.2 (SPSS, Chicago, Illinois, USA).

\section{RESULTS}

\section{Patient characteristics}

\section{Erosive reflux disease}

Epigastric pain (100.0\%), epigastric discomfort (100.0\%), regurgitation $(81.1 \%)$, heart burn $(18.6 \%)$ and chest pain $(9.3 \%)$ were the predominant symptoms of ERD at pretreatment. These symptoms interfered with daily activities in $39.5 \%$ of the ERD patients. The dietary patterns of the patients with ERD included overeating (65.1\%), heavy eating (62.7\%), eating at night $(60.5 \%)$, drinking soda $(34.9 \%)$, salty and spicy food $(18.6 \%)$ and greasy food $(18.6 \%)$.

\section{Non-erosive reflux disease}

Epigastric pain (100.0\%), epigastric discomfort (100.0\%), regurgitation $(73.5 \%)$ and heart burn $(2.9 \%)$ were the predominant symptoms of NERD at pretreatment. These symptoms interfered with the daily activities in $23.5 \%$ of NERD patients. The dietary patterns for NERD included overeating (64.6\%), heavy eating $(50.0 \%)$, eating at night $(44.1 \%)$, greasy food $(38.2 \%)$, salty and spicy food (38.2\%) and drinking soda (32.4\%).

\section{Grouping}

Erosive reflux disease

A total of 43 ERD patients (20 males, 23 females) were enrolled. The mean age was $11.5 \pm 3.2$ years (range $5.0-18.0$ )

\section{Table 1 The Los Angeles classification of esophagitis}

Grade A One (or more) mucosal break no longer than $5 \mathrm{~mm}$ that does not extend between the tops of two mucosal folds

Grade B One (or more) mucosal break more than $5 \mathrm{~mm}$ that does not extend between the tops of two mucosal folds

Grade C One (or more) mucosal break that is continuous between the tops of two or more mucosal folds but which involves less than $75 \%$ of the circumference

Grade D One (or more) mucosal break which involves at least $75 \%$ of the oesophageal circumference 
and the mean body mass index (BMI) was $18.8 \pm 3.4 \mathrm{~kg} / \mathrm{m}^{2}$ (table 2). Six patients that refused to take the medicine for more than 2 weeks because of improved symptoms were lost to follow-up. After 8 weeks of treatment, 37 patients were assessed for changes in symptoms and divided into three subgroups: $6(16.2 \%)$ patients in the 'symptoms-resolved' group, $22(59.5 \%)$ patients in the 'symptoms-attenuated' group and 9 $(24.3 \%)$ patients in the 'symptoms-persistent' group (figure 1). There were no statistically significant differences among the groups with regard to age and BMI (table 2).

\section{Non-erosive reflux disease}

A total of 34 NERD patients ( 9 males, 25 females) were enrolled. The mean age was $12.0 \pm 4.1$ years (range $4.0-17.0$ ) and the mean BMI was $18.3 \pm 3.1 \mathrm{~kg} / \mathrm{m}^{2}$ (table 2). Fourteen patients that refused to take the medicine for more than 2 weeks because of improved symptoms were lost to follow-up. After 8 weeks of treatment, 20 patients were assessed for a change of symptoms and were divided into three groups: 7 (35.0\%) patients in the 'symptoms-resolved' group, 10 (50.0\%) patients in the 'symptoms-attenuated' group and $3(15.0 \%)$ patients in the 'symptoms-persistent' group (figure 2). There were no statistically significant differences among the groups with regard to age and BMI (table 2).

\section{Assessment of symptoms}

Erosive reflux disease

Six patients showed complete improvement of symptoms at 8 weeks. Lansoprazole was discontinued in the "symptomsresolved' patients. All of these improved patients $(100 \%)$ remained improved at 16 and 24 weeks (figure 1). Twentytwo patients in the 'symptoms-attenuated' group were maintained with 'on-demand' therapy. Sixteen (72.7\%) patients 'improved' at 16 weeks and $18(81.8 \%)$ patients at 24 weeks ( $p=0.487$ vs 16 weeks). The nine patients in the 'symptomspersistent' group were maintained on PPI treatment without dose reduction for an additional 16 weeks. Six (66.7\%) patients 'improved' at 16 and 24 weeks. Among the six patients that refused further treatment because of symptom improvement

Table 2 Demographic characteristics at baseline

\begin{tabular}{|c|c|c|c|c|c|c|}
\hline & Total & Symptoms-resolved & Symptoms-attenuated & Symptoms-persistent & No more treatment & ${ }^{*}$ p Value \\
\hline ERD (N) & 43 & 6 & 22 & 9 & 6 & \\
\hline $\operatorname{Sex}(M / F)$ & $20: 23$ & $3: 3$ & $14: 8$ & $2: 7$ & $1: 5$ & 0.075 \\
\hline Age (year) & $11.5 \pm 3.2$ & $10.3 \pm 3.2$ & $11.1 \pm 3.0$ & $12.0 \pm 3.8$ & $12.0 \pm 2.5$ & 0.469 \\
\hline (Min-max) & $(5.0-18.0)$ & $(7.0-15.0)$ & $(5.0-16.0)$ & $(6.0-18.0)$ & $(9.0-15.0)$ & \\
\hline $\mathrm{BMI}\left(\mathrm{kg} / \mathrm{m}^{2}\right)$ & $18.8 \pm 3.4$ & $19.3 \pm 3.0$ & $19.3 \pm 3.7$ & $19.0 \pm 3.2$ & $15.8 \pm 2.1$ & 0.462 \\
\hline (Min-max) & $(13.3-27.7)$ & $(15.8-22.9)$ & $(14.9-27.6)$ & $(15.8-19.8)$ & $(13.3-18.7)$ & \\
\hline NERD (N) & 34 & 7 & 10 & 3 & 14 & \\
\hline $\operatorname{Sex}(M / F)$ & $9: 25$ & $3: 4$ & $2: 8$ & $1: 2$ & $3: 11$ & 0.725 \\
\hline Age (year) & $12.0 \pm 4.1$ & $14.1 \pm 2.7$ & $11.3 \pm 4.2$ & $12.0 \pm 3.6$ & $11.5 \pm 4.6$ & 0.509 \\
\hline (Min-max) & $(4.0-17.0)$ & $(9.0-17.0)$ & $(5.0-17.0)$ & $(8.0-15.0)$ & $(4.0-17.0)$ & \\
\hline $\mathrm{BMI}\left(\mathrm{kg} / \mathrm{m}^{2}\right)$ & $18.3 \pm 3.1$ & $18.8 \pm 1.6$ & $17.9 \pm 3.0$ & $16.5 \pm 2.5$ & $19.0 \pm 3.7$ & 0.656 \\
\hline (Min-max) & $(14.1-30.0)$ & $(16.5-21.7)$ & $(14.2-24.9)$ & $(14.1-19.0)$ & $(15.3-30.0)$ & \\
\hline
\end{tabular}

${ }^{*} p$ Value among subgroups.

$\mathrm{BMI}$, body mass index; ERD, erosive reflux disease; NERD, non-erosive reflux disease.

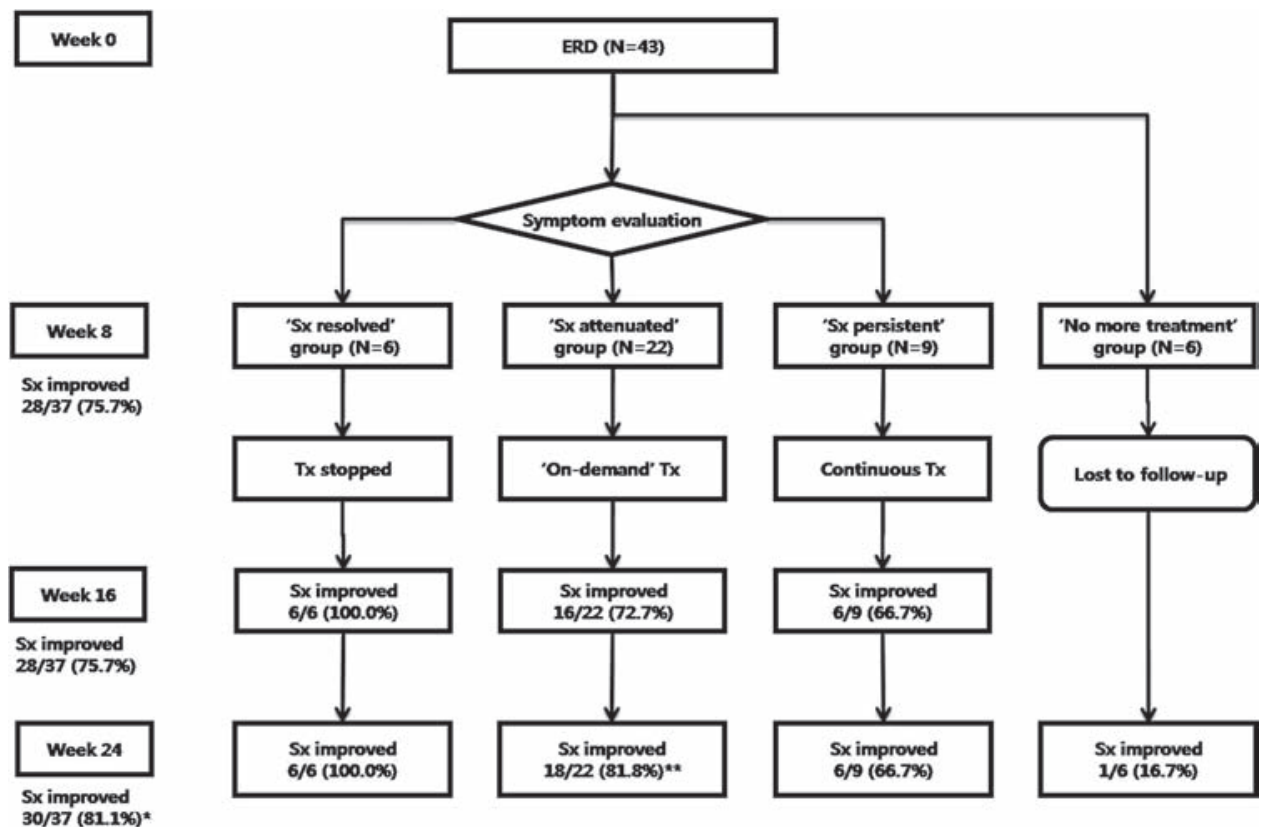

Figure 1 Schematic flow of the study for ERD. ${ }^{*} p=0.487$, compared with week 16 ; ${ }^{*} p=0.487$, compared with week 16 . ERD, erosive reflux disease; Sx, symptom; Tx, treatment. 


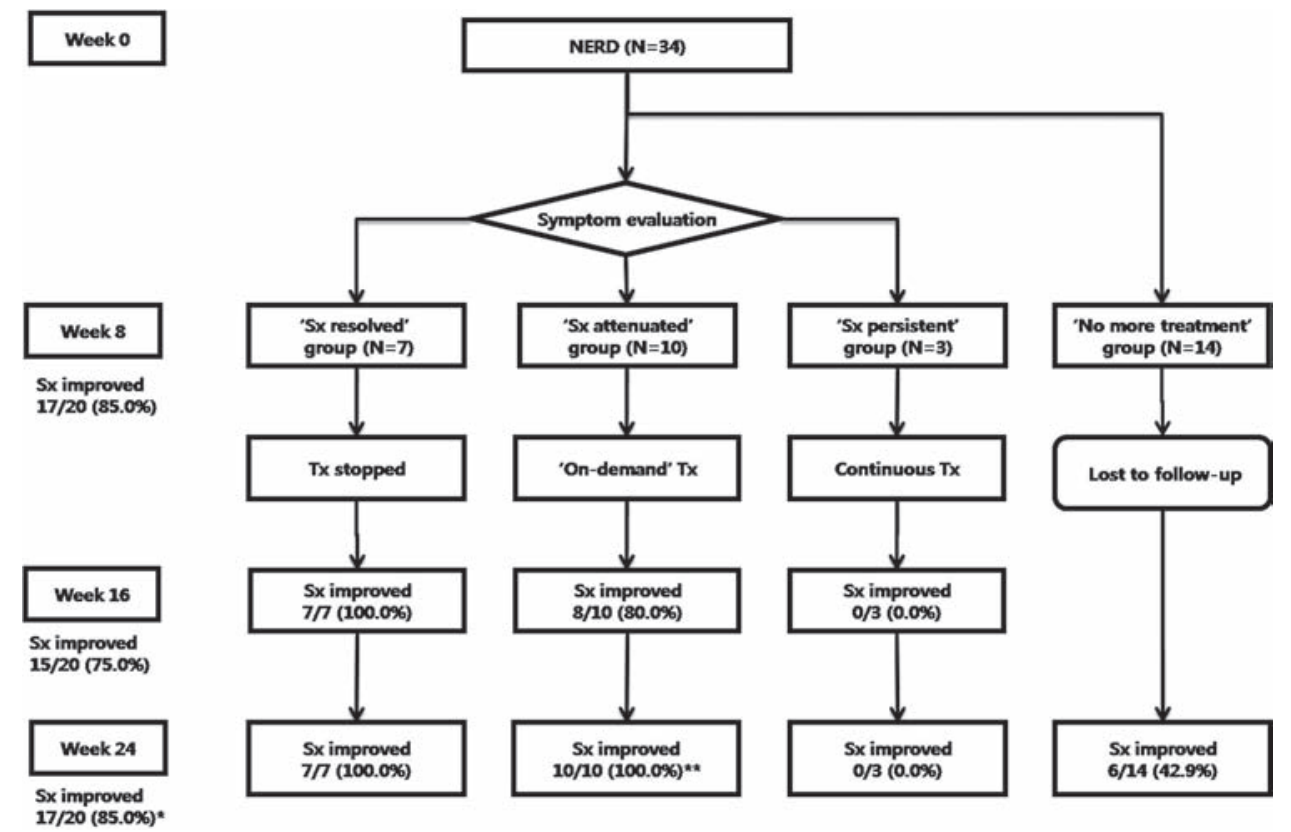

Figure 2 Schematic flow of the study for NERD. ${ }^{*} p=0.163$, compared with week $16 ;{ }^{*} p=0.157$, compared with week 16 . NERD, non-erosive reflux disease; Sx, symptom; Tx, treatment.

('no more treatment' group), symptoms recurred in five (83.3\%) patients at 24 weeks. In summary, 28 (75.7\%) out of 37 subjects had improvement of symptoms at 8 weeks, 28 $(75.7 \%)$ at 16 weeks and $30(81.1 \%)$ at 24 weeks ( $p=0.487$ vs 16 weeks).

\section{Non-erosive reflux disease}

Seven patients showed complete improvement at 8 weeks. Lansoprazole was discontinued in these 'symptoms-resolved' patients. All of them (100\%) remained improved atweeks 16 and 24 (figure 1). Ten patients in the 'symptoms-attenuated' group were maintained with 'on-demand' therapy. Eight $(80.0 \%)$ patients 'improved' at 16 weeks and 10 (100.0\%) patients remained 'improved' at 24 weeks ( $p=0.157$ vs 16 weeks). Three patients in the 'symptoms-persistent' group were maintained on PPI treatment without dose reduction for an additional 16 weeks. None of them improved at 16 and 24 weeks. Of the 14 patients that refused further treatment because of symptom improvement ('no more treatment' group), symptoms recurred in eight $(57.1 \%)$ patients at 24 weeks. In summary, $17(85.0 \%)$ out of 20 subjects had improved symptoms at 8 weeks, 15 $(75.0 \%)$ at 16 weeks and $17(85.0 \%)$ at 24 weeks ( $p=0.163$ vs 16 weeks).

\section{Endoscopy}

Initial endoscopy

Endoscopies with biopsies were performed in 43 patients with ERD and 34 patients with NERD. Twenty-five (58.1\%) out of 43 patients with ERD had a grade A reflux esophagitis as defined by the Los Angeles classification, 18 (41.9\%) out of 43 were grade $B$; there was no patient with a severe esophagitis over grade $\mathrm{C}$.

Follow-up endoscopy after 8 weeks of treatment

Follow-up endoscopies after 8 weeks of treatment were performed in 14 patients with ERD. The initial endoscopies revealed that $4(28.6 \%)$ out of 14 patients had grade $A$ and $10(71.4 \%)$ grade B. Follow-up endoscopies after 8 weeks revealed that 8 (57.1\%) out of 14 were grade A and 6 (42.9\%) grade $\mathrm{B}$. Comparing the initial endoscopic findings with the follow-up results, 7 (50.0\%) out of 14 improved, 6 (42.9\%) out of 14 showed no change in symptoms and 1 (7.1\%) out of 14 deteriorated.

\section{Adverse events}

Among the 57 patients (ERD 37, NERD 20) that were treated with lansoprazole over 2 months, three (5.3\%) patients had adverse events; hair loss developed in a child with ERD at 4 months, chest tightness was found in a child with NERD at 2 weeks and lower abdominal pain was present in a child with NERD at 4 months. Further medication was discontinued and the side effects resolved.

\section{DISCUSSIONS}

This is the first paediatric study on long-term PPI treatment strategies including 'on-demand' treatment and continuous treatment without dose reduction. The results of this study show that when the medication was discontinued in the 'symptoms-resolved' patients with ERD or NERD at 8 weeks, all of the patients remained improved at week 24 , without any additional medication. The ERD patients in the 'symptomsattenuated' group at 8 weeks showed improvement in $72.7 \%$ at week 16 and in $81.1 \%$ at week 24 with an 'on-demand' treatment strategy. For NERD patients, improvement was achieved in $80 \%$ at week 16 and in $100 \%$ at week 24 . The ERD patients in the 'symptoms-persistent' group showed improvement in $66.7 \%$ at weeks 16 and 24 , respectively. These findings suggest that the symptom evaluation at 8 weeks was an important factor for successful long-term treatment and that 16 weeks of therapy was adequate for long-term treatment compared to 24 weeks of therapy.

Six patients in the ERD group and 14 patients in the NERD group refused more treatment after 2 weeks of lansoprazole therapy because their symptoms resolved; they remained improved in only $16.7 \%$ of the ERD patients and $42.9 \%$ of the 
NERD patients at 24 weeks. Based on the above findings, it is suggested that at least 8 weeks of treatment and symptom assessment after this treatment period are necessary for adequate treatment of reflux esophagitis in children.

In the literature, 'on-demand' therapy has been reported to be more effective with regard to the cost-effectiveness of long-term management of GERD.${ }^{17}$ Especially in patients with NERD, some investigators have reported that 'on-demand' therapy is sufficient to control the symptoms and continuous therapy is needed only in cases with severe esophagitis. ${ }^{17} 18$ Bytzer et a ${ }^{19}$ recommended that NERD be treated with a PPI at the standard dose for 4-8 weeks, and more treatment with other drugs or 'on-demand' therapy as needed. However, most studies of 'on-demand' treatment included adult cases; there has been no report on paediatric 'on-demand' therapy in patients with reflux esophagitis. Therefore, the results of this study have significance in that the 'on-demand' treatment strategy was compared with other treatment strategies for the first time in children and it had equal efficacy.

Adverse events associated with treatment were reported in three cases $(3 \%)$ out of 57 patients that were treated for over 2 months. The side effects included hair loss, lower abdominal pain and chest tightness. Three adverse events were considered to be mild and improved after discontinuation of the medication. This rate of complication was lower than reported in other lansoprazole studies. ${ }^{11} 20$

The limitations of this study include the following. This study was not randomised or controlled. The number of subjects in each group was relatively small. Biopsy was not performed at follow-up endoscopy; therefore, we were not able to determine the correlation between symptoms and histological severity.

In conclusion, the results of this study suggest three alternatives including 'on-demand' therapy for the long-term treatment of GERD after 8 weeks of PPI therapy in children. The findings showed that 8 weeks of therapy was effective. In addition, the selection of which alternative to use for long-term management was successfully determined according to the symptom assessment at 8 weeks. 'On-demand' therapy was equally effective. Finally, the results showed that 16 weeks of therapy had the same efficacy as 24 weeks of therapy with regard to long-term PPI treatment.

\section{Competing interests None.}

Patient consent Obtained.

Ethics approval Approval for the study was obtained from the Institutional Review Board at Samsung Medical Center.

Provenance and peer review Not commissioned; externally peer reviewed.

\section{REFERENCES}

1. Vandenplas $\mathbf{Y}$, Rudolph CD, Di Lorenzo C, et al. Pediatric gastroesophageal reflux clinical practice guidelines: joint recommendations of the North American Society for Pediatric Gastroenterology, Hepatology, and Nutrition (NASPGHAN) and the European Society for Pediatric Gastroenterology, Hepatology, and Nutrition (ESPGHAN). J Pediatr Gastroenterol Nutr 2009;49:498-547.

2. Sherman PM, Hassall E, Fagundes-Neto U, et al. A global, evidence-based consensus on the definition of gastroesophageal reflux disease in the pediatric population. Am J Gastroentero/ 2009;104:1278-95; quiz 1296.

3. Dent J, Brun J, Fendrick A, et al. An evidence-based appraisal of reflux disease management-the Genval workshop report. Gut1999;44:S1-16.

4. Fock KM, Talley NJ, Fass R, et al. Asia-Pacific consensus on the management of gastroesophageal reflux disease: update. J Gastroenterol Hepatol 2008;23:8-22.

5. Vakil N, van Zanten SV, Kahrilas P, et al. The Montreal definition and classification of gastroesophageal reflux disease: a global evidence-based consensus. Am J Gastroenterol 2006;101:1900-20; quiz 1943.

6. Giannini EG, Zentilin P, Dulbecco P, et al. Management strategy for patients with gastroesophageal reflux disease: a comparison between empirical treatment with esomeprazole and endoscopy-oriented treatment. Am J Gastroenterol 2008;103:267-75.

7. Kusano M, Shimoyama Y, Kawamura 0, et al. Proton pump inhibitors improve acid-related dyspepsia in gastroesophageal reflux disease patients. Dig Dis Sci 2007; 52:1673-7.

8. Mine S, lida T, Tabata T, et al. Management of symptoms in step-down therapy of gastroesophageal reflux disease. J Gastroenterol Hepatol 2005;20:1365-70.

9. Domínguez-Muñoz JE, Sobrino M. Clinical response (remission of symptoms) in erosive and non-erosive gastro-oesophageal reflux disease. Drugs 2005;65 1:43-50

10. Bak YT. Management strategies for gastroesophageal reflux disease. J Gastroenterol Hepatol 2004;19:S49-53.

11. Fiedorek S, Tolia V, Gold BD, et al. Efficacy and safety of lansoprazole in adolescents with symptomatic erosive and non-erosive gastroesophageal reflux disease. J Pediatr Gastroenterol Nutr 2005;40:319-27.

12. Orenstein SR. Pediatric erosive esophagitis maintenance: finally, some level 1 evidence! Am J Gastroentero/ 2007;102:1298-300.

13. Cezard JP. Managing gastro-oesophageal reflux disease in children. Digestion 2004;69:3-8.

14. Boccia G, Manguso F, Miele E, et al. Maintenance therapy for erosive esophagitis in children after healing by omeprazole: is it advisable? Am J Gastroenterol 2007; 102:1291-7.

15. Lee TJ, Fennerty MB, Howden CW. Systematic review: Is there excessive use of proton pump inhibitors in gastro-oesophageal reflux disease? Aliment Pharmacol Ther 2004:20:1241-51.

16. Armstrong D, Bennett JR, Blum AL, et al. The endoscopic assessment of esophagitis: a progress report on observer agreement. Gastroenterology 1996;111:85-92

17. Talley NJ, Venables TL, Green JR, et al. Esomeprazole $40 \mathrm{mg}$ and $20 \mathrm{mg}$ is efficacious in the long-term management of patients with endoscopy-negative gastro-oesophageal reflux disease: a placebo-controlled trial of on-demand therapy for 6 months. Eur J Gastroenterol Hepatol 2002;14:857-63.

18. Leodolter A, Penagini R. On-demand therapy is a valid strategy in GERD patients: pros and cons. Dig Dis 2007;25:175-8.

19. Bytzer P. Goals of therapy and guidelines for treatment success in symptomatic gastroesophageal reflux disease patients. Am J Gastroenterol 2003;98:S31-9.

20. Reimer C, Bytzer P. Clinical trial: long-term use of proton pump inhibitors in primary care patients - a cross sectional analysis of 901 patients. Aliment Pharmacol Ther 2009;30:725-32. 